\title{
Personalidad y apoyo social como predictores de la añoranza del hogar en estudiantes universitarios
}

\section{Personality and social support as predictors of homesickness in college students}

\author{
Gustavo Francisco Medina Bolívar ${ }^{1}$ y Carlos Enrique Zerpa ${ }^{2}$
}

\begin{abstract}
Resumen
El objetivo del presente estudio fue evaluar el poder predictivo de los rasgos de personalidad y las dimensiones del apoyo social percibido sobre la añoranza del hogar en estudiantes universitarios. Se trata de una investigación no experimental, de campo y transversal correlacional-causal, realizada con una muestra de 228 estudiantes universitarios, hombres y mujeres, con edades comprendidas entre los 16 y 25 años que cursaban el primer periodo académico en universidades públicas y privadas de la ciudad de Caracas, Venezuela. Para la evaluación de las variables se administró la Escala de Añoranza del Hogar de Archer y colaboradores, el Inventario de Personalidad de Goldberg y el Inventario de Apoyo Social Percibido de Dunn y colaboradores. Para analizar los resultados se emplearon estadísticos descriptivos, correlaciones y análisis de regresión múltiple. Se encontró que la añoranza del hogar puede predecirse inversamente a partir de las variables de apoyo de amigos, el neuroticismo y el apoyo religioso; el apego al hogar, por las variables de neuroticismo y apoyo religioso, y el desagrado por la universidad, también inversamente, por el apoyo de amigos y el neuroticismo.
\end{abstract}

Palabras clave: Añoranza del hogar; Personalidad; Apoyo social; Estudiantes universitarios

\section{SUMMARY}

The aim of the present study was to assess the predictive power of personality traits and perceived social support on homesickness in college students. The study would be characterized as non-experimental, transactional, fieldwork and transversal correlational-causal. A total of 228 college students aged 16 to 25 years, in their first academic period in public and private universities of Caracas, Venezuela participated. Measurements included the Homesickness Scale by Archer et al., Goldberg's Personality Inventory and the Perceived Social Support Inventory by Dunn et al. Data analyses included descriptive statistics, correlations, and multiple regression. Results indicate that homesickness can be inversely predicted by friends support, neuroticism and religious support variables; attachment to home, by neuroticism and religious support variables. Dislike for the University was predicted, also inversely, by friends support and neuroticism.

Key words: Homesickness; Personality; Social support; College students.

\footnotetext{
${ }^{1}$ Instituto de Psicología, Universidad Central de Venezuela, Av. Neverí, Centro Comercial Los Chaguaramos, Piso 2, Oficina 2-10, Urb. Los Chaguaramos, 1040 Caracas, Venezuela, tel. (58)21-26-05-28-30, correo electrónico: gustavo.f.medina@ucv.ve. Artículo recibido el 13 de febrero y aceptado el 16 de mayo de 2017.

${ }^{2}$ Departamento de Ciencia y Tecnología del Comportamiento, Universidad Simón Bolívar, Edificio de Estudios Generales, Piso 1, Oficina 111-D, Valle de Sartenejas, 89000 Baruta, Edo. Miranda, Venezuela, tel. (58)21-29-06-38-13, correo electrónico: czerpa@usb.ve.
} 


\section{INTRODUCCIÓN}

$\mathrm{D}$ esde la antigüedad, los seres humanos han observado posiblemente que la lejanía del hogar, el distanciamiento con las figuras parentales y algunos pares significativos ocasionan cambios en el comportamiento y emociones tales como tristeza, angustia y añoranza hacia las personas y el entorno que se han dejado atrás. La literatura clásica y obras como las de Matt (2007) así lo sugieren. No fue sino hasta mediados del siglo XIX cuando comienza a estudiarse este fenómeno entre los combatientes europeos, mismo que ha sido denominado "añoranza del hogar" (homesick$n e s s)^{3}$ y que fue definido por Thurber y Walton (2007) como la "angustia y deterioro funcional causado por una separación real o anticipada del hogar y de los objetos de apego, tales como los padres" (p. 192).

Se ha observado que dicho fenómeno ocurre en casi cualquier entorno en el que las personas se separan real o imaginariamente de sus figuras y entornos significativos, pero se ha estudiado de manera sistemática en niños de educación primaria, en jóvenes que asisten a campamentos, en militares que han sido trasladados lejos de sus lugares de origen y, principalmente, en estudiantes adolescentes y adultos tempranos (de entre 17 y 23 años de edad) que recién ingresan a una institución universitaria alejada de su hogar (Eurelings-Bontekoe, Brouwers, Verschuur y Duijsens, 1998; Matthews y Deary, 1998). Algunos de los investigadores que han llevado a cabo trabajos sobre la añoranza del hogar (Brewin, Furnham y Howes, 1989; Eurelings-Bontekoe et al., 1998; Malaklolunthu y Sateyen, 2011; Matt, 2007; Shal, Sharbaf, Abdekhodaee, Masoleh y Salehi, 2011; Shin y Abell, 1999; Tartakovsky, 2007; Thurber, 2005) han encontrado una amplia caracterización y asociación con variables psicosociales y de personalidad.

En este sentido, la respuesta de los estudiantes a la añoranza del hogar está muy posiblemente relacionada con el apoyo social, que ha demostrado ser de gran valor para reducir el malestar asociado a muchas condiciones diferentes (Brewin et al., 1989). Se trata de una variable ampliamente

${ }^{3}$ El término en español aquí empleado es la traducción más exacta posible del citado vocablo inglés (N. del E.). estudiada en el área de la psicología de la salud, y diversos autores coinciden acerca de su papel moderador sobre los efectos de los trastornos psicosociales y, en particular, en las respuestas ante el estrés y la ansiedad. A este respecto, Brewin et al. (1989) señalan la necesidad de conocer "en qué medida los estudiantes con añoranza del hogar confiarían su experiencia a los demás, y en qué medida buscarían a otras personas en lugar de aislarse socialmente" (p. 469).

Asimismo, distintos autores han estudiado las relaciones entre los cinco grandes factores de la personalidad (Costa y Mc Crae, 1992; Goldberg, 1993) y la añoranza del hogar, hallando asociaciones positivas entre la misma y el neuroticismo (Eurelings-Bontekoe et al., 1998; Van Tilburg, Vingerhoets y Van Heck, 1999), lo que plantea la necesidad de llevar a cabo investigaciones que hagan posible proporcionar una base empírica de las relaciones propuestas entre los rasgos de personalidad disfuncional (como por ejemplo el neuroticismo) y la añoranza del hogar.

En los últimos años se ha podido apreciar cómo la escala de neuroticismo se ha vinculado a muy diversas afecciones físicas y psicológicas, entre ellas la añoranza del hogar (Brewin et al., 1989; Eurelings-Bontekoe et al., 1998; Ferraz y Pereira, 2002; Khademi y Farshi, 2013; Van Tilburg et al., 1999; Verschuur y Eurelings-Bontekoe, 2003).

El referido fenómeno ha sido igualmente estudiado en países cuyas condiciones socioeconómicas y educativas permiten el intercambio de estudiantes extranjeros bajo la figura de becas; en otros casos, ocurre que estudiantes del mismo país se trasladan a otras ciudades a cursar estudios superiores, como ocurre por ejemplo en el Reino Unido, Holanda, Canadá, Estados Unidos, China y Turquía, por solo mencionar algunos, observándose una incidencia de la añoranza del hogar que va de 19 a 91\% (Eurelings-Bontekoe et al., 1998; Matthews y Deary, 1998). No obstante, los estudios sobre la añoranza del hogar y las variables asociadas a esta son escasos en los países latinoamericanos, y en particular en Venezuela. A propósito de ello, Flett, Endler y Besser (2009) señalan que deberían realizarse investigaciones en muestras de estudiantes universitarios que provengan de diferentes culturas, incluyendo, de ser posible, 
jóvenes y adultos. Por consiguiente, al ser este un fenómeno escasamente estudiado en la región, se disponen de escasas evidencias empíricas de su efecto en estudiantes universitarios de habla hispana y de estudios que relacionen de manera conjunta los rasgos de personalidad y las dimensiones del apoyo social percibido con la añoranza del hogar; dada la relevancia e impacto social que tendría el esclarecimiento de estas relaciones a propósito del proceso de adaptación al entorno universitario, surge la necesidad de llevar a cabo una investigación con estudiantes foráneos venezolanos, alumnos de tres instituciones de educación universitaria ubicadas en el Distrito Metropolitano de Caracas. Por lo anterior, se planteó conocer el efecto predictivo de la personalidad y el apoyo social en la añoranza del hogar en estudiantes universitarios.

\section{MÉTODO}

\section{Tipo y diseño de investigación}

De acuerdo a la clasificación de Kerlinger y Lee (2002), la presente investigación fue de carácter no experimental y de campo, con un diseño transversal correlacional-causal que, de acuerdo a Hernández, Fernández y Baptista (2010), permite describir relaciones entre dos o más variables en un momento determinado, ya sea en términos correlacionales o en función de la relación de causa-efecto, lo que posibilita predecir el comportamiento de una variable a partir de otras, denominadas predictoras.

\section{Participantes}

La muestra, recolectada a partir de un procedimiento de tipo no probabilístico e intencional, estuvo conformada por 228 estudiantes, $43.86 \%$ de hombres y $56.14 \%$ de mujeres, con edades comprendidas entre 16 y 25 años de edad $(\mathrm{M}=17.54$; D.E. $=1.28)$, que cursaban el primer periodo académico de carreras largas en tres instituciones universitarias, de las cuales $87.72 \%$ eran públicas y $12.28 \%$ privadas, ubicadas en el Distrito Metropolitano de Caracas: Universidad Simón Bolívar (49.93\% de los estudiantes), Universidad Central de Venezuela (40.79\%) y Universidad Santa María $(12.28 \%)$.

\section{Instrumentos}

Para la realización del estudio se emplearon los siguientes instrumentos de recolección de información:

Cuestionario Big Five (Goldberg, 1993), en versión adaptada y validada en Venezuela por Saputi (2008). Se trata de un cuestionario de autopercepción, de lápiz y papel, que se responde en aproximadamente diez minutos, que permite evaluar las dimensiones propuestas por la teoría de Goldberg y que consta de cinco dimensiones: Extroversión, Agradable (afabilidad), Apertura a la experiencia, Concienzudo (conciencia) y Neuroticismo. Se encuentra conformado por 40 ítems en forma de adjetivos que se valoran mediante una escala de respuesta tipo Likert de cinco puntos, (de 1, "No es cierto, casi nunca soy de esa manera", a 5, "Totalmente, soy así casi siempre". En la muestra venezolana que empleó Saputi (2008), con edades comprendidas entre 18 y 60 años, los coeficientes alfa de Cronbach arrojaron valores de .70 a .84 , lo que indica una alta consistencia interna.

Inventario de Apoyo Social (Dunn, Putallaz, Sheppard y Lindstrom, 1987), en versión adaptada y validada en Venezuela por Angelucci (2011). Se trata de un instrumento de lápiz y papel que se responde en un tiempo aproximado de diez minutos y que mide tres factores que hacen referencia a fuentes de apoyo social percibido: Apoyo de amigos, Apoyo familiar y Apoyo religioso. Consta de 28 ítems y contiene una escala de respuestas tipo Likert de cuatro puntos cuyos valores van de 0 , "Nada", a 3, "Mucho". La confiabilidad obtenida por Angelucci (2011) fue de .85 para la prueba total, de. 857 para Apoyo de amigos, de .80 para Apoyo familiar y de .76 para Apoyo religioso.

Homesickness Scale (Archer, Ireland, Amos, Broad y Currid, 1998), adaptada a universitarios venezolanos para el presente estudio. Es un inventario de lápiz y papel diseñado para medir la añoranza del hogar. Puede responderse en un tiempo aproximado de diez minutos. La escala adaptada consta de 27 ítems que se califican mediante una escala de respuestas tipo Likert de cuatro puntos: de 1, "Completamente en desacuerdo", a 4, "Completamente de acuerdo". Consta de dos dimensiones: Desagrado por la universidad, conformada por 14 ítems, y Apego al hogar, con 13. La escala mostró valores de consistencia interna ob- 
tenida mediante el coeficiente alfa de Cronbach de .872 para la escala total, de .836 para la dimensión Desagrado por la universidad y de .826 para la dimensión Apego al hogar, lo que indica una adecuada confiabilidad.

Adicionalmente a estos instrumentos, se empleó una hoja de datos sociodemográficos e información personal de los participantes, la cual permitió la verificación de los criterios para ser incluidos en la muestra de estudio, tales como edad, sexo, lugar de procedencia, periodo académico cursado, institución educativa, carrera, experiencias previas de estar lejos del hogar, tiempo transcurrido lejos del hogar al momento de la recolección de datos, antecedentes de enfermedad mental y uso de medicamentos al momento del estudio.

Asimismo, se empleó un formato de consentimiento informado que recogió los aspectos éticos de la investigación y en el que se expusieron los objetivos de la investigación, los posibles riesgos, la voluntariedad para participar y abandonar el estudio cuando así lo desearan y otras consideraciones éticas incluidas en el Código de Ética del Psicólogo Venezolano y en el de la American Psychological Association. Solo se consideraron las personas que leyeron por completo el citado formato y que lo firmaron, accediendo así a participar de manera voluntaria en la investigación.

\section{Procedimiento}

La aplicación de los instrumentos se llevó a cabo tras el permiso concedido por las autoridades académicas para acceder a las aulas en los horarios de clase de los estudiantes del primer periodo académico en las diferentes carreras de cada universidad. Se acudió a cada grupo de clase y se informó sobre las características del estudio, el propósito y la manera en que sería manejada la información recopilada. Solo participaron en la investigación aquellos estudiantes que provenían del interior del país y que firmaron el formato de consentimiento informado indicado antes. Los criterios de inclusión empleados fueron, a saber: que tuvieran una edad comprendida entre los 16 y 25 años, que cursaran el primer periodo académico en la institución universitaria, que su hogar o lugar de graduación estuviese ubicado fuera del Distrito Metropolitano de Caracas y que no sufriera enfermedades menta- les ni que estuviera recibiendo medicamentos para estas en los dos últimos años.

Una vez recolectada la información, los datos fueron transcritos, codificados y analizados a partir de los estadísticos descriptivos, correlaciones y análisis de regresión múltiple.

\section{RESULTADOS}

Para el logro del objetivo de la investigación fue necesario describir primeramente las variables de estudio en la población, conocer las relaciones entre las variables, verificar los supuestos para la aplicación de análisis multivariantes de datos $\mathrm{y}$, finalmente, estimar las regresiones múltiples para conocer la importancia relativa de los rasgos de personalidad y las dimensiones del apoyo social percibido como predictores de la añoranza del hogar y sus dimensiones en la muestra seleccionada de estudiantes universitarios.

En la Tabla 1 se muestran los resultados descriptivos para las variables bajo estudio.

En el caso de la Añoranza del hogar total y de la dimensión de Apego al hogar, los estudiantes mostraron puntajes moderados, pero bajos en el caso de la dimensión Desagrado por la universidad. Puede observarse que, al comparar los puntajes entre hombres y mujeres, se obtuvieron diferencias significativas entre los grupos, mostrando las mujeres puntajes medios superiores a los obtenidos por los hombres, aunque con valores moderados en el caso de la Añoranza del hogar total y Apego al hogar, y moderados o bajos en Desagrado por la universidad. En los rasgos de personalidad no se observaron diferencias significativas $(p<.01)$ entre los puntajes medios de hombres y mujeres, y a excepción de la dimensión Agradable, cuya media se ubicó en valores bajos respecto al rango, el resto de las dimensiones medidas mostraron valores más bien moderados (apertura y neuroticismo) y moderados o altos (Extroversión y Concienzudo). Con relación a las dimensiones del apoyo social percibido, puede observarse que los participantes mostraron en general puntajes altos respecto al rango observado, a excepción de la dimensión Apoyo religioso, en que los puntajes se concentraron más hacia los valores moderados. Cabe destacar que en el caso del apoyo social se pudieron 
Tabla 1. Estadísticos descriptivos de las variables consideradas.

\begin{tabular}{|l|r|r|r|r|r|c|c|}
\hline \multicolumn{1}{|c|}{ Variable } & $\begin{array}{c}\text { Media } \\
\text { mujeres }\end{array}$ & $\begin{array}{c}\text { Media } \\
\text { hombres }\end{array}$ & \multicolumn{1}{c|}{$\boldsymbol{t}$} & $\begin{array}{c}\text { Media } \\
\text { muestral }\end{array}$ & \multicolumn{1}{c|}{ D.E. } & $\begin{array}{c}\text { Rango } \\
\text { posible }\end{array}$ & $\begin{array}{c}\text { Rango } \\
\text { obtenido }\end{array}$ \\
\hline Añoranza del hogar total & 52.922 & 46.880 & $4.272^{*}$ & 50.27 & 10.992 & $27-108$ & $30-82$ \\
\hline Desagrado por la universidad & 20.992 & 18.590 & $3.678^{*}$ & 19.94 & 5.482 & $14-56$ & $13-35$ \\
\hline Apego al hogar & 25.781 & 22.420 & $4.533^{*}$ & 24.31 & 5.790 & $13-52$ & $12-40$ \\
\hline Extroversión & 21.406 & 21.010 & 0.630 & 21.23 & 4.786 & $6-30$ & $7-30$ \\
\hline Agradable & 18.223 & 19.290 & -1.792 & 18.69 & 4.467 & $8-40$ & $9-32$ \\
\hline Concienzudo & 36.188 & 34.760 & 2.013 & 35.56 & 5.349 & $9-45$ & $16-45$ \\
\hline Apertura & 3.758 & 31.850 & -1.429 & 31.24 & 5.739 & $9-45$ & $17-45$ \\
\hline Neuroticismo & 21.953 & 21.130 & 1.183 & 21.59 & 5.217 & $8-40$ & $8-38$ \\
\hline Apoyo de amigos & 24.461 & 22.800 & 2.065 & 23.73 & 6.069 & $0-33$ & $5-33$ \\
\hline Apoyo familiar & 18.711 & 17.250 & $3.576^{*}$ & 18.07 & 2.985 & $0-21$ & $2-21$ \\
\hline Apoyo religioso & 5.023 & 3.910 & $2.760^{*}$ & 4.54 & 3.067 & $0-12$ & $0-12$ \\
\hline
\end{tabular}

* Significativo a $p<.01$

apreciar diferencias estadísticamente significativas $(p<.01)$ en Apoyo religioso y en Apoyo familiar, donde las mujeres alcanzaron medias superiores.

Como procedimiento previo al análisis de regresión, se calcularon las correlaciones producto-momento de Pearson entre las variables de la investigación con el propósito de conocer cuáles fueron las relaciones más significativas entre las variables. En la Tabla 2 puede apreciarse que la única dimensión de la personalidad que se relacionó con puntuaciones bajas, positivas y estadísticamente significativa (a un nivel de $p=.01$ ) con la añoranza del hogar y sus dimensiones de fue
Neuroticismo, con valores de $r=.213$ en Añoranza del hogar total, $r=.172$ en Desagrado por la universidad y $r=.205$ en Apego al hogar. Asimismo, puede observarse que en las dimensiones de Apoyo social percibido, las de Apoyo de amigos $(r=-.278)$ y Apoyo familiar $(r=-.188)$ mostraron asociaciones bajas, negativas y estadísticamente significativas con el Desagrado por la universidad $(p=.01)$, mientras que la dimensión de Apoyo religioso mostró asociaciones bajas, positivas y significativas con la dimensión de Apego al hogar $(r=.204)$.

Tabla 2. Correlaciones bivariadas entre las variables de estudio.

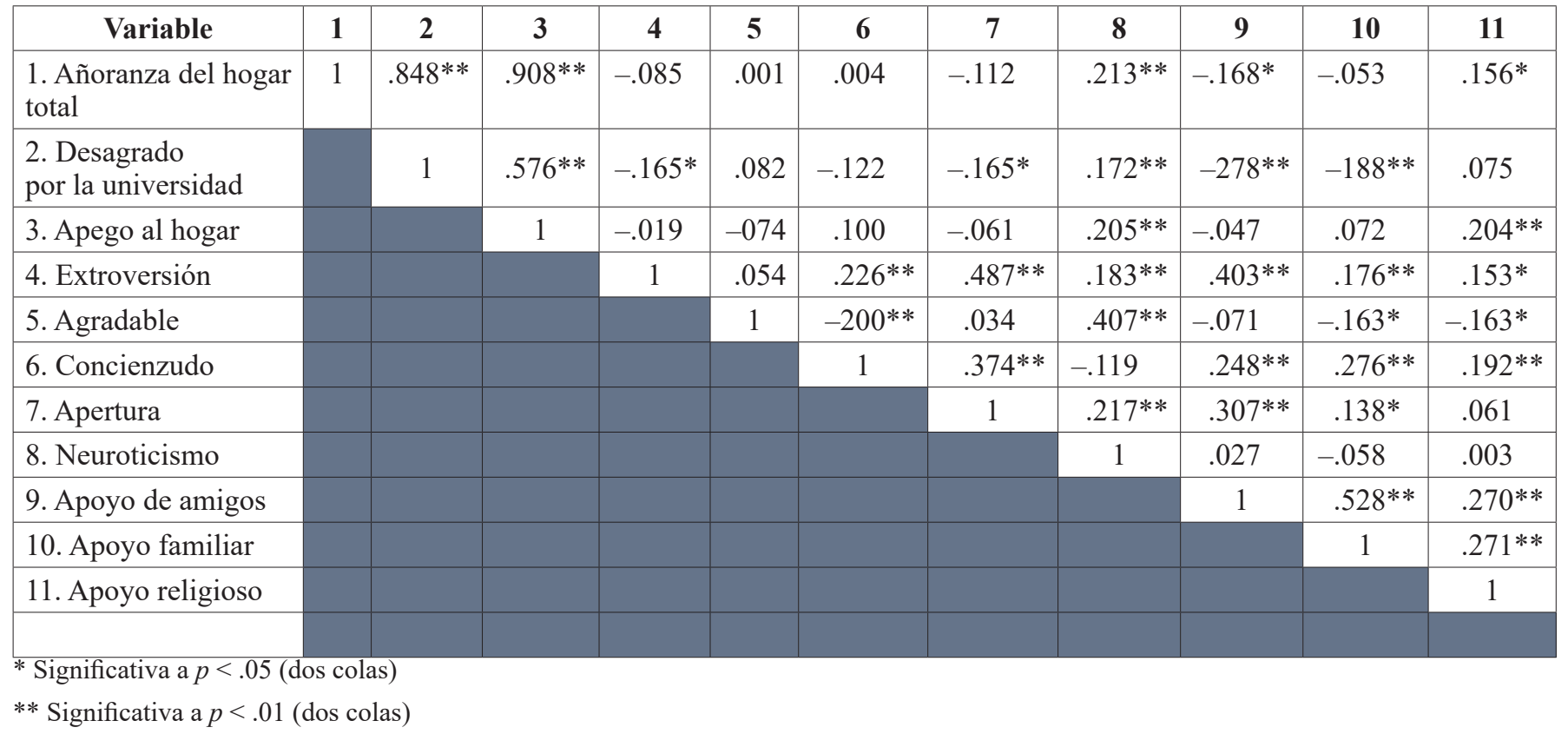


Posterior al análisis de las correlaciones, se comprobaron los supuestos propuestos por Hair, Anderson, Tatham y Black (1999) para la realización del análisis de regresión múltiple, a saber: normalidad a través de la prueba de Kolmogorov-Smirnov; linealidad a través de la inspección visual de los gráficos de regresión parcial; homocedasticidad a partir de la inspección visual de los gráficos de los residuales; independencia de los términos de error analizando los valores de la prueba de Durbin-Watson y mediante la inspección visual de los gráficos de los residuos, y ausencia de multicolinealidad entre las variables independientes a partir de la inspección visual de la matriz de correlaciones de las variables.

Se pudieron comprobar los supuestos, a excepción del supuesto de normalidad en las dimensiones Desagrado por la universidad de Añoranza del hogar, y Apoyo familiar y Apoyo religioso del Apoyo social percibido. A este respecto, Hair et al. (1999) sugieren que una forma solventar dicho incumplimiento es mediante la transformación de las variables, ya sea a través del logaritmo, de la raíz cuadrada o incluso del inverso de la variable. Se realizaron los cálculos necesarios tomando como base lo propuesto, pero no se observaron mejoras significativas en los resultados, razón por la cual se optó por dejar las regresiones encontradas y tomar con cautela los resultados obtenidos.

Para llevar a cabo la regresión por el método paso a paso, fue necesario fijar previamente el criterio de probabilidad de entrada (que permite identificar y evitar que la información proporcionada por dos variables sea redundante) y el criterio de probabilidad de salida del modelo. De esta manera, se partió de un criterio de entrada de las variables de $p<.01$ y un criterio de salida de $p>.05$. Este análisis se condujo estudiando cada componente de la variable dependiente por separado: primero Añoranza del hogar total, luego Apego al hogar y finalmente para Desagrado por la universidad.

\section{Añoranza del hogar total}

En la Tabla 3 se muestran los resultados obtenidos luego de calcular la regresión paso a paso entre todas las variables independientes (dimensiones de la personalidad y del apoyo social percibido) y la añoranza del hogar total.

Tabla 3. Regresión entre las variables independientes y añoranza del hogar total.

\begin{tabular}{|c|c|c|c|c|c|c|c|c|c|}
\hline \multirow[b]{2}{*}{ Modelo } & \multirow[b]{2}{*}{ Variables independientes } & \multicolumn{8}{|c|}{ Variable dependiente: Añoranza del hogar total } \\
\hline & & $\boldsymbol{\beta}$ & $\mathbf{t}$ & $p$ & $\mathbf{R}$ & $\mathbf{R}^{2}$ & $\begin{array}{c}\mathrm{R}^{2} \\
\text { ajustado }\end{array}$ & F & Sig. \\
\hline 1 & Neuroticismo & .213 & 3.279 & $.001 *$ & 0.213 & 0.045 & 0.041 & 10.75 & $.001 *$ \\
\hline \multirow{2}{*}{2} & Neuroticismo & .218 & 3.397 & $.001 *$ & 0.275 & 0.076 & 0.067 & 9.20 & $.000^{*}$ \\
\hline & Apoyo de amigos & -.174 & -2.711 & $.007 *$ & & & & & \\
\hline \multirow{3}{*}{3} & Neuroticismo & .219 & 3.490 & $.001 *$ & 0.346 & 0.120 & 0.108 & 10.180 & $.000 *$ \\
\hline & Apoyo de amigos & -.233 & -3.577 & $.000 *$ & & & & & \\
\hline & Apoyo religioso & .219 & 3.359 & $.001 *$ & & & & & \\
\hline
\end{tabular}

Se puede observar que la añoranza del hogar es mejor explicada por el tercer modelo, en el que puede apreciarse que existe una correlación múltiple moderada-baja $(\mathrm{R}=.346)$ entre Neuroticismo, Apoyo de amigos y Apoyo religioso con Añoranza del hogar, que en su conjunto explican $12 \%$ de la varianza total $\left(\mathrm{R}^{2}=.120\right)$ y que es significativa desde el punto de vista estadístico $(\mathrm{F}=10.18$; $\mathrm{gl}=3 ; p=.000)$. Es importante señalar que la combinación lineal de las variables incluidas en este modelo de predicción deja de explicar $88 \%$ de la varianza de la Añoranza del hogar total (coeficiente de no determinación $=.880$ ).

Para conocer el aporte que cada una de las variables predictoras del modelo tiene individualmente sobre la Añoranza del hogar total, se puede observar en la tabla anterior que las variables cuyas aportaciones al modelo de predicción resultaron estadísticamente significativas con un nivel de significación de $\alpha=.01$ fueron las de Neuroticismo, Apoyo de amigos y Apoyo religioso, y asimismo que las variables Neuroticismo y Apoyo 
religioso aportan a la predicción una correlación positiva, baja y significativa, mientras que la variable Apoyo de amigos aporta una correlación negativa, baja y significativa.

La ecuación de regresión resultante fue la siguiente:

$\mathrm{Yi}=46.776+(.219) * \mathrm{X} 1+(-.233) * \mathrm{X} 2+(.219) * \mathrm{X} 3+1.381$

\section{Apego al hogar}

Respecto a la dimensión Apego al hogar, puede decirse que la combinación lineal de las variables independientes que mejor predice la dimensión es la reportada en el segundo modelo (Tabla 4).

Tabla 4. Regresión entre las variables independientes y la dimensión apego al hogar.

\begin{tabular}{|c|l|c|c|c|c|c|c|c|c|}
\hline \multirow{2}{*}{ Modelo } & \multirow{2}{*}{$\begin{array}{c}\text { Variables } \\
\text { independientes }\end{array}$} & \multicolumn{6}{|c|}{ Variable dependiente: Apego al hogar } \\
\cline { 3 - 11 } & & $\boldsymbol{t}$ & $\boldsymbol{p}$ & $\mathbf{R}$ & $\mathbf{R}^{\mathbf{2}}$ & $\begin{array}{c}\mathbf{R}^{\mathbf{2}} \\
\text { ajustado }\end{array}$ & $\mathbf{F}$ & \multirow{2}{*}{ Sig. } \\
\hline 1 & Neuroticismo & .205 & 3.150 & .002 & .205 & .042 & .038 & 9.925 & .002 \\
\hline \multirow{2}{*}{2} & Neuroticismo & .205 & 3.204 & .002 & .289 & .083 & .075 & 10.245 & .000 \\
\cline { 2 - 10 } & Apoyo religioso & .203 & 3.188 & .002 & & & & & \\
\hline
\end{tabular}

En la tabla puede apreciarse una correlación múltiple baja $(\mathrm{R}=.289)$ entre Neuroticismo y Apoyo religioso, que en conjunto explica $8.3 \%$ de la varianza total de Apego al hogar, significativa desde el punto de vista estadístico. Es importante señalar que esta combinación lineal de las variables deja de explicar $91.7 \%$ de la varianza de Apego al hogar (coeficiente de no determinación $=.917$ ).

Para conocer el aporte individual de cada una de las variables predictoras sobre el apego al hogar, se puede observar asimismo en la anterior tabla que las variables cuyas aportaciones al modelo de predicción resultaron estadísticamente significativas, con un nivel de significación de.01, fueron las variables Neuroticismo y Apoyo religioso, que aportaron asociaciones positivas y bajas. La ecuación de regresión resultante fue como sigue:

$$
\mathrm{Yi}=17.664+(0.205) * \mathrm{X} 1+(.203) * \mathrm{X} 2+5.568
$$

\section{Desagrado por la universidad}

Con relación a la dimensión Desagrado por la universidad de Añoranza del hogar, se obtuvieron dos modelos a través de la regresión paso a paso, siendo el segundo de ellos el que explicó el mayor porcentaje de varianza. En la Tabla 5 se puede observar que en ese modelo hubo una correlación múltiple moderada-baja entre Neuroticismo y Apoyo de amigos, que en conjunto explican $11 \%$ de la varianza total (coeficiente de determinación = .110), significativa desde el punto de vista estadístico. Es importante señalar que la combinación lineal de las variables incluidas en este modelo de predicción deja de explicar $89 \%$ de la varianza de Desagrado por la universidad (coeficiente de no determinación $=.890$ ).

Tabla 5. Regresión entre las variables independientes y la dimensión Desagrado por la universidad.

\begin{tabular}{|c|c|c|c|c|c|c|c|c|c|}
\hline \multirow[b]{2}{*}{ Modelo } & \multirow{2}{*}{$\begin{array}{c}\text { Variables } \\
\text { independientes }\end{array}$} & \multicolumn{8}{|c|}{ VD: Desagrado por la universidad } \\
\hline & & $\boldsymbol{\beta}$ & $\mathbf{t}$ & $p$ & $\mathbf{R}$ & $\mathbf{R}^{2}$ & $\begin{array}{c}R^{2} \\
\text { ajustado }\end{array}$ & $\mathbf{F}$ & Sig. \\
\hline 1 & Apoyo de amigos & -.278 & -4.348 & .000 & .278 & .077 & .007 & 18.906 & .000 \\
\hline \multirow{2}{*}{2} & Apoyo de amigos & -.283 & -4.493 & .000 & .331 & 0.11 & .102 & 13.851 & .000 \\
\hline & Neuroticismo & .180 & 2.863 & .005 & & & & & \\
\hline
\end{tabular}

Explorando las asociaciones individuales de las variables predictoras sobre la dimensión Desagrado por la universidad, se puede observar en la tabla que la variable Apoyo de amigos aporta a la predicción una correlación negativa, baja y significa- tiva, y la variable Neuroticismo una correlación positiva, baja y significativa. La ecuación de regresión resultante fue la siguiente:

$$
Y_{i}=21.789+(-.283) * \mathrm{X} 1+(.180) * \mathrm{X} 2+4.870
$$




\section{DISCUSIÓN}

La presente investigación tuvo como objetivo principal predecir la añoranza del hogar a partir de los rasgos de personalidad y las dimensiones del apoyo social percibido. Primeramente, se caracterizó la muestra en función a las variables de estudio, observándose puntuaciones que se ubicaron hacia los valores promedio de Añoranza del hogar y sus dimensiones. En este sentido, se esperaba que la muestra puntuara niveles altos en mayor proporción que la encontrada debido a que su mayor parte se encontraba en el periodo en que, según la literatura (Eurelings-Bontekoe et al., 1998; Matthews y Deary, 1998), la añoranza del hogar resulta sorprendentemente común entre estudiantes universitarios y se estima una incidencias que oscila entre 39 y $72 \%$, siendo mayor en los casos de China, Turquía y Estados Unidos (entre 19 y 95\%).

Dada la baja incidencia de la añoranza del hogar en la muestra de estudio, cabe mencionar que Dawson y Pooley (2013) sostienen que el apoyo social percibido contribuye a que las personas puedan adaptarse más fácilmente en la transición hacia la universidad, encontrándose relaciones negativas con disfunciones tales como ansiedad, soledad y depresión. En ese mismo orden de ideas, Martínez et al. (2014) destacan el papel del apoyo social percibido como un factor protector importante ante las experiencias y demandas que implica la transición a la universidad.

Dado que la muestra de estudio estuvo conformada predominantemente por mujeres $(56.14 \%)$, se calcularon los estadísticos de contraste pertinentes, comprobándose diferencias estadísticamente significativas a favor de las mismas. Los hallazgos de la presente investigación relativos al sexo son contrarios a los de Fisher y Hood (1988) y Verschuur y Eurelings-Bontekoe (2003), quienes no encontraron diferencias estadísticamente significativas entre la prevalencia o la intensidad de la añoranza del hogar y el sexo. No obstante, tales resultados pudieran ir de la mano con los de Ferraz y Pereira (2002), que encontraron que las mujeres tendieron a puntuar niveles altos de añoranza del hogar, aunque no de un modo estadísticamente significativo. Lo aquí encontrado se relaciona con los hallazgos de Bethelmy y Guarino (2008) en su estudio con médicos venezola- nos, en el que las mujeres tuvieron una tendencia mayor a manifestar sintomatología física y psicológica, lo cual las autoras relacionan con el rol de madres o cuidadoras asociado a las mujeres y a las tareas del hogar.

En la dimensión Apego al hogar, las mujeres alcanzaron puntuaciones por encima de las obtenidas por los hombres. Tales resultados fueron significativos en el presente estudio, aunque en investigaciones como la de Flett et al. (2009), a pesar de haberse encontrado medias ligeramente superiores en las mujeres estudiantes universitarias que conformaron su muestra respecto a la de los hombres, dichas diferencias no fueron significativas en el caso de estos últimos. Ferraz y Pereira (2002) sostienen que las estudiantes mujeres tienden a ser más dependientes, nostálgicas y afectivas respecto de sus familias.

La prevalencia vista en las mujeres pudiera relacionarse con las estrategias de afrontamiento que emplean los estudiantes. A este respecto, Matud (2004) señala que las mujeres muestran mayores niveles de afrontamiento emocional y evitación que los hombres, mientras que estos últimos emplean predominantemente estrategias de afrontamiento racional, de desapego e inhibición emocional, por lo que en el caso de los presentes hallazgos, el estilo de afrontamiento podría estar influenciando las relaciones encontradas en función del sexo.

En cuanto a las dimensiones de personalidad, se pudieron apreciar valores más bien moderados en la muestra y sin diferencias estadísticamente significativas en cuanto al sexo. Se esperaba que las diferencias encontradas en las dimensiones de Neuroticismo, Concienzudo y Extroversión fueran estadísticamente significativas, y que estuvieran en consonancia con lo reportado por Ferraz y Pereira (2002), en cuyo estudio las mujeres obtuvieron puntuaciones medias superiores y estadísticamente significativas en la escala de neuroticismo.

De acuerdo con los resultados obtenidos en las diferentes dimensiones del apoyo social percibido, los estudiantes pertenecientes a la muestra de estudio lograron puntajes moderados-altos en cuanto al Apoyo de amigos, predominantemente altos en Apoyo familiar, y moderados-bajos en Apoyo religioso. A este respecto, Urani, Miller, Johnson y Petzel (2003) encontraron que el apoyo 
social en el ambiente del hogar de las personas antes de ir a la universidad tiene una relación positiva y estadísticamente significativa con el apoyo social en el medio educativo, por lo que ese elevado apoyo familiar percibido por la totalidad de la muestra estudiada pudo deberse a las experiencias de apoyo previas a su ingreso a la universidad, fomentadas en aquellos estudiantes que puntuaron alto.

Los presentes resultados fueron contrastados en función del sexo, observándose que las mujeres mostraron medias superiores a la de los hombres en las tres dimensiones descritas, pero solo fueron significativas estadísticamente en los casos de Apoyo social y Apoyo religioso. Las diferencias observadas en los promedios obtenidos en las dimensiones de apoyo social en función del sexo difieren de las encontradas por Rosa-Rodríguez, Negrón, Maldonado, Quiñonez y Toledo (2015), quienes no hallaron diferencias estadísticamente significativas en el apoyo social percibido en función al sexo; sin embargo, coinciden con los resultados de Feldman et al. (2008), en cuyo estudio las mujeres tuvieron una mayor percepción del apoyo social que los hombres. Barra (2011) atribuye tales diferencias a factores de socialización y a los roles de género, añadiendo que mientras que los hombres ponen un mayor énfasis en la autonomía, independencia y autoconfianza, las mujeres tienden a ser formadas para ser más dependientes, con mayor conciencia de sus problemas y mayor disposición para la búsqueda de la ayuda de los demás.

En cuanto a las principales relaciones surgidas entre la añoranza del hogar y las variables de personalidad, se apreció que el neuroticismo mostró asociaciones con las dimensiones Desagrado por la universidad y Apego al hogar, lo cual se corresponde con lo encontrado por Khademi y Farshi (2013); en su estudio con estudiantes universitarios, estos autores encontraron una correlación baja, positiva y significativa entre el neuroticismo y la añoranza del hogar, y también con lo hallado por Ferraz y Pereira (2002), quienes a su vez descubrieron una relación positiva, baja y significativa entre las variables mencionadas. De esta manera, en la muestra de estudiantes evaluada, aquellos que exhibieron una menor adaptación emocional - caracterizada por pesimismo, ansiedad, nerviosismo y tensión- tendieron a reportar puntuaciones mayores en la añoranza del hogar en general.
A propósito de las relaciones encontradas entre el neuroticismo y la añoranza del hogar, es importante señalar que cuando esta última es persistente, recurrente y pasa de ser aguda a crónica, puede exacerbar diversas patologías. Si bien es cierto que no genera trastornos mentales, es posible que su cronicidad y falta de atención oportuna profundicen ciertos trastornos de la personalidad o patologías de base que en ocasiones ni el mismo individuo conoce, lo que repercute en su salud y en las relaciones que mantiene con su entorno. A este respecto, Stroebe, Schut y Nauta (2015) propusieron un modelo en el que se considera la añoranza del hogar como una especie de "miniduelo", que se asemeja a las consideraciones teóricas de la añoranza del hogar valoradas con el instrumento empleado en el presente estudio para su medición: problemas referidos al hogar (apego al hogar), por una parte, y problemas referidos al nuevo entorno (desagrado por la universidad) por la otra, el cual proponen someter a valoración empírica en investigaciones futuras y cuyas aportaciones ofrecen alternativas para su prevención y tratamiento. Eurelings-Bontekoe et al. (1998) hallaron asimismo una marcada prevalencia de desórdenes de personalidad en personas que reportaron padecer o haber padecido añoranza del hogar, con diferencias estadísticamente significativas respecto a quienes no padecían ni habían padecido la misma.

En cuanto a las relaciones encontradas entre la añoranza del hogar y las dimensiones del apoyo social percibido, los estudiantes que percibían contar con un apoyo mayor de las agrupaciones religiosas y sociales a las que pertenecían tendieron a mostrar puntajes más elevados de apego al hogar, mientras que aquellos que contaban con el apoyo de sus familiares, amigos y pares, mostraron puntajes más bajos de desagrado por la universidad.

Van Tilburg, Vingerhoets y Van Heck (1997) afirman que los estudiantes con un considerable apoyo social son menos propensos a padecer añoranza del hogar que aquellos que han perdido el apoyo social; Barra (2011), a su vez, señala que quienes cuentan con el apoyo de la familia y los amigos muestran menores índices de depresión $\mathrm{y}$ ansiedad, lo que les permite afrontar mejor los problemas del día a día. Por ende, los resultados van en la misma dirección que lo planteado por la literatura existente hasta el momento. 
Sobre el apoyo social existe una amplia bibliografía que lo relaciona con los procesos de salud y enfermedad. De hecho, se ha encontrado que el apoyo social percibido, tal como el que puede provenir de la familia extendida (hermanos, profesores, compañeros, etc.), se ha considerado como una contribución benéfica a la adaptación positiva de la transición a la universidad (Urquant y Pooley, 2007), y que cuando en los semestres iniciales los estudiantes forman o se unen a grupos de apoyo social en los primeros semestres de su carrera, adquieren más fácilmente las habilidades para hacer frente a su entorno (Poyrazly y López, 2007). Se han propuesto algunos modelos, como el DPM (Dual Process Model of Coping with Bereavement) de Stroebe y Schut (1999), que brindan alternativas para explicar esas relaciones (cf. Sun, 2015).

Finalmente, para alcanzar el objetivo de evaluar el poder predictivo de las diferentes dimensiones de la personalidad y el apoyo social percibido sobre el añoranza del hogar en una muestra de estudiantes, fue necesario computar los modelos de regresión múltiple a través del método paso a paso, que mostró que las variables de neuroticismo y apoyo religioso correlacionaron baja y positivamente con la añoranza del hogar total, mientras que el apoyo de amigos lo hizo de forma baja, negativa y significativa desde el punto de vista estadístico, por lo que pareciera que las personas que perciben contar con el apoyo de sus asociaciones religiosas, que poseen rasgos neuróticos y que tienen una baja percepción del apoyo de sus amigos y pares, tienden a manifestar mayores niveles de añoranza del hogar.

Vale la pena mencionar que investigaciones como las de Poyrazly y López (2007) y Urquant y Pooley (2007) han destacado el papel amortiguador del apoyo social como factor protector ante la añoranza del hogar. Sin embargo, en el presente caso la dimensión del apoyo religioso tuvo un efecto diferente del esperado. Se presume que ello pudo deberse a que, en general, las asociaciones de índole religiosa y social tienden a fomentar espacios para la autorrevelación ante los otros, así como el contacto cognitivo y emocional consigo mismo. Es posible que tal contacto contribuya a que las personas que se encuentran lejos del hogar tiendan a tener pensamientos persistentes y recu- rrentes acerca de su familia, amigos y personas significativas que permanecieron en el lugar donde vivía. No obstante, esas hipótesis deben someterse a contrastación.

El modelo obtenido para la dimensión de Apego al hogar solo explicó 8.3\% de la varianza no atribuible al error o al azar, a través de la combinación lineal de las variables Neuroticismo y Apoyo religioso, cada una de las cuales aportó relaciones bajas, positivas y estadísticamente significativas. Puede decirse con la debida cautela que aquellos estudiantes que presentan rasgos de personalidad caracterizados por la tendencia a exhibir mala adaptación emocional, pesimismo, ansiedad, nerviosismo, tristeza y tensión, y que además perciben que cuentan con el apoyo de las agrupaciones religiosas, sociales y deportivas a las que pertenecen, muestran mayores posibilidades de manifestar preocupaciones, pensamientos y sueños relacionados con el hogar, pérdida del sí mismo y evitación.

La dimensión Desagrado por la universidad fue mejor predicha por la combinación lineal de Apoyo de amigos y Neuroticismo, las cuales en su conjunto explicaron $11 \%$ de la varianza total no atribuible al error o al azar, apreciándose que el primero tuvo una correlación negativa baja y significativa, mientras que el segundo se relacionó de forma positiva, baja y significativa. A propósito de Apoyo de amigos, estas últimas relaciones encontradas en los modelos derivados del método por pasos pudiera explicarse mediante la teoría de la pertenencia, cuya premisa es que los seres humanos poseen una tendencia innata a mantener relaciones interpersonales duraderas. Watt y Badger (2009), basados en esta teoría, hallaron una relación positiva y significativa entre la necesidad de pertenecer y la añoranza del hogar, encontrando además que las personas que se sentían aceptadas en la comunidad mostraban una menor añoranza del hogar, lo cual ocurría independientemente de la cantidad de amigos que tuviesen, por lo que en el caso de la presente investigación sería la sensación de sentirse aceptados en la comunidad universitaria lo que determinó haber experimentado o no la añoranza del hogar.

Cabe mencionar en este punto que si bien las referencias en la literatura existente son vastas en los países anglosajones, en su mayoría relacio- 
nan la añoranza del hogar con la personalidad (Brewin et al., 1989; Eurelings-Bontekoe et al., 1998; Ferraz y Pereira, 2002; Khademi y Farshi, 2013; Van Tilburg et al., 1999; Verschuur y Eurelings-Bontekoe, 2003) y con el apoyo social (Poyrazly y López, 2007; Urani et al., 2003; Urquant y Pooley, 2007). En la literatura temática no se obtuvieron reportes de investigaciones en las que se relacionara la añoranza del hogar, la personalidad y el apoyo social de manera conjunta, y menos aún en cuanto a sus dimensiones constitutivas.

Lo que se ha observado es que la mayor parte de las investigaciones reportadas emplean los puntajes globales en lugar de hacerlo por dimensiones, sobre todo en el caso de la añoranza del hogar y del apoyo social; por ende, son escasas las evidencias empíricas de que se dispone para contrastar los modelos de predicción encontrados, y la presente investigación se constituye como un aporte novedoso que contribuirá a ampliar el conocimiento en el estudio de esa variable.

En conclusión, las asociaciones significativas halladas fueron bajas y directas entre la añoranza del hogar total y el neuroticismo y entre el apego al hogar y el apoyo religioso, y bajas e indirectas entre el desagrado por la universidad y el apoyo de amigos y entre el desagrado por la universidad y el apoyo familiar.

Puede afirmarse, entonces, que la añoranza del hogar puede ser predicha por el neuroticismo, el apoyo religioso y el apoyo de amigos, siendo mayor en estudiantes con rasgos neuróticos de personalidad que perciben que cuentan con el apoyo de las organizaciones religiosas y sociales a las que se pertenecen y que no perciben carecer del de sus amigos y pares.
Por su parte, el apego al hogar pudo predecirse por el neuroticismo y el apoyo religioso, pues los estudiantes mostrarán mayor apego al hogar mientras más agudos sean sus rasgos de personalidad neurótica y mayor su percepción del apoyo proveniente de las asociaciones religiosas y sociales a las que pertenecen.

Finalmente, el desagrado por la universidad puede predecirse por el apoyo de amigos y el neuroticismo: aquellos estudiantes que mostraron mayores rasgos de neuroticismo y que tuvieron una baja percepción del apoyo de sus amigos y pares mostraron un mayor desagrado por la universidad.

Debido al muestreo empleado para el presente estudio y el bajo poder explicativo de los modelos obtenidos, los resultados deben ser tomados con reserva debido a la posible influencia de factores que atentan contra su validez. Futuras investigaciones pueden llevarse a cabo con procedimientos de muestreo y diseños de investigación más robustos que garanticen la validez de los resultados.

El presente estudio representa, en fin, un aporte al conocimiento de que se dispone acerca de la variable y da lugar a profundizar o contrastar los hallazgos, e incluso ampliarlos analizando las posibles relaciones con variables tales como estilo de afrontamiento, tipo de apego, resiliencia, percepción de control, ansiedad y sensibilidad emocional, que si bien han sido estudiadas de manera individual en su relación con la añoranza del hogar, se desconoce la aportación conjunta de las mismas en la predicción del referido fenómeno en muestras similares.

\section{REFERENCIAS}

Angelucci, L. (2011). Emoción y salud: un modelo causal. Tesis inédita de doctorado. Caracas: Universidad Central de Venezuela.

Archer, J., Ireland, J., Amos, S., Broad, H. y Currid, L. (1998). Derivation of a homesickness scale. British Journal of Psychology, 89, 205-221. doi: 10.1177/0013916510361872.

Barra, E. (2011). Influencia de la autoestima y del apoyo social percibido sobre el bienestar psicológico de estudiantes universitarios chilenos. Diversitas: Perspectivas en Psicología, 8(1), 29-38. Disponible en línea: http://www.redalyc.org/articulo. oa?id=67923973002.

Bethelmy R., L. y Guarino, L. (2008). Estrés laboral, afrontamiento, sensibilidad emocional y síntomas físicos y psicológicos en médicos venezolanos. Revista Colombiana de Psicología, 17, 43-58. Disponible en línea: http://www.redalyc.org/articulo. oa?id=80411803003. 
Brewin, C., Furnham, A. y Howes, M. (1989). Demographic and psychological determinants of homesickness and confiding among students. British Journal of Psychology, 8, 467-477. doi: 10.1111/j.2044-8295.1989.tb02336.x.

Costa, P. y Mc Crae, R. (1992). Professional manual for the NEO-PI-R and NEO-FFI. Odessa, FL: Psychologycal Assessment Resources.

Dawson, M. y Pooley, J. (2013). Resilience: The role of optimism, perceived parental autonomy and perceived social support in first year students. Journal of Education and Training Studies, 1(2), 38-49. doi: https://doi.org/10.11114/jets.v1i2.137.

Dunn, S., Putallaz, M., Sheppard, B. y Lindstrom, R. (1987). Social support and adjustment in gifted adolescents. Journal of Educational Psychology, 79(4), 467-473. doi: 10.1037/0022-0663.79.4.467.

Eurelings-Bontekoe, E., Brouwers, E., Verschuur, M. y Duijsens, I. (1998). DSM-III-R and ICD-10 personalitydisorder features among women experiencing two types of self-reported homesickness: An exploratory study. British Journal of Psychology, 89, 405-416. doi: 10.1111/j.2044-8295.1998.tb02693.x.

Feldman, L., Goncalves, L., Chacón-Pignau, G., Zaragoza, J., Bagés, N. y de Pablo, J. (2008). Relaciones entre estrés académico, apoyo social, salud mental y rendimiento académico en estudiantes universitarios venezolanos. Universitas Psychologica, 7(3), 739-751. Disponible en línea: http://www.uacm.kirj.redalyc.redalyc.org/articulo.oa?id=64770311.

Ferraz, M. y Pereira, A. (2002). A dinâmica da personalidade e homesickness (saudades de casa) dos jóvenes estudiantes universitarios. Psicología, Saúde y Doencas, 3(2), 149-164. Disponible en línea: http:/www.uacm.kirj.redalyc.redalyc.org/ articulo.oa? id=36230204.

Fisher, S. y Hood, B. (1988). Vulnerability factors in the transition to university: Self-reported mobility history and sex differences as factors in psychological disturbance. British Journal of Psychology, 79, 309-320. doi: 10.1111/j.2044-8295.1988. tb02290.x.

Flett, G., Endler, N. y Besser, A. (2009). Separation anxiety, perceived controllability, and homesickness. Journal of Applied Social Psychology, 39(2), 265-282. doi: 10.1111/j.1559-1816.2008.00438.x.

Goldberg, L. (1993). The estructure of phenotypic personality traits. American Psychologist, 48(1), 26-34.

Hair, J., Anderson, R., Tatham, R. y Black, W. (1999). Análisis multivariante. Madrid: Prentice-Hall.

Hernández, R., Fernández, C. y Baptista, P. (2010). Metodología de la investigación (5a ed.). México: McGraw-Hill.

Kerlinger, F. y Lee, H. (2002). Investigación del comportamiento. México: McGraw-Hill/ Interamericana.

Khademi, A. y Farshi, A. (2013). The role of personality traits and resilience on homesickness of college students. Procedia Social and Behavioral Sciences, 82(3), 537-541. doi: 10.1016/j.sbspro.2013.06.305.

Malaklolunthu, S. y Sateyen, P. (2011). Adjustment problems among international students in Malaysian private higher education institutions. Procedia Social and Behavioral Sciences, 15, 833-837. doi: 10.1016/j.sbspro.2011.03.194.

Martínez L., Z., Páramo, M., Guisande, M., Tinajero, C., da Silva, L. y Rodríguez, M. (2014). Apoyo social en universitarios españoles de primer año: Propiedades psicométricas del Social Support Questionnaire-Short Form y la Social Provision Scale. Revista Latinoamericana de Psicología, 46(2), 102-110. doi: 10.1016/S0120-0534(14)70013-5.

Matt, S. (2007). A hunger for home: Homesickness and food in a global consumer society. The Journal of American Culture, 30(1), 6-17. doi: 10.1111/j.1542-734X.2007.00461.x.

Matthews, G. y Deary, I. (1998). Personality traits. Cambridge, MA: Cambridge University Press.

Matud, P. (2004). Gender differences in stress and coping styles. Personality and Individual Differences, 37, 1401-1415. doi: 10.1016/j.paid.2004.01.010.

Poyrazly, S. y López, M. (2007). An exploratory study of perceived discrimination and homesickness: A comparison of international students and American students. The Journal of Psychology: Interdisciplinary and Applied, 141(3), 263-280. doi: 10.3200/JRLP.141.3.263-280.

Rosa-Rodríguez, Y., Negrón, N., Maldonado, Y., Quiñonez, A. y Toledo, N. (2015). Dimensiones de bienestar psicológico y apoyo social percibido con relación al sexo y nivel de estudio en universitarios. Avances en Psicología Latinoamericana, 33(1), 31-43. doi: dx.doi.org/10.12804/apl33.01.2015.03.

Saputi, D. (2008). Big Five, estilos de manejo de conflicto y resultados de la negociación, un análisis de ruta. Tesis inédita de maestría. Caracas: Universidad Simón Bolívar.

Shal, R., Sharbaf, H., Abdekhodaee, M., Masoleh, S. y Salehi, I. (2011). Survey the relationship between attachment style and general self efficacy with homesickness among college students. Procedia Social and Behavior Sciences, 3, 538-541. doi: 10.1016/j.sbspro.2011.10.105.

Shin, H. y Abell, N. (1999). The Homesickness and Contentment Scale: Developing a culturally sensitive measure of adjustment for Asian. Research on Social Work Practice, 9(1), 40-45. doi: 10.1177/104973159900900104.

Stroebe M.S. y Schut, H. (1999). The Dual Process Model of Coping with Bereavement: Rationale and description. Death Studies, 23, 197-224. doi: 10.1080/074811899201046.

Stroebe, M., Schut, H. y Nauta, M. (2015). Is homesickness a mini-grief? Development of a dual process model. Clinical Psychological Science, 4(2), 344-358. doi: 10.1177/2167702615585302. 
Sun, J. (2015). Homesickness at college: A predictive model for first-time students. Unpublished Doctoral Thesis. Ames, IO: Iowa State Institute.

Tartakovsky, E. (2007). A longitudinal study of acculturative stress and homesickness: High-schoool adolescents immigrating from Russia and Ukraine to Israel without parents. Social Psychiatryc and Psychiatric Epidemiology, 42(6), 485-494. doi: 10.1007/s00127-007-0184-1.

Thurber, C. (2005). Multimodal homesickness prevention in boys spending 2 weeks at a residential summer camp. Journal of Consulting and Clinical Psychology, 73(3), 555-560. doi: 10.1037/0022-006X.73.3.555.

Thurber, C. y Walton, E. (2007). Preventing and treating homesickness. Child and Adolescent Psychiatric Clinics of North America, 16(4), 843-858. doi:10.1016/j.chc.2007.05.003.

Urani, M., Miller, S., Johnson, J. y Petzel, T. (2003). Homesickness in socially anxious first year college students. College Student Journal, 37(3), 392. Disponible en línea: http://www.campspirit.com/files/2012/05/HS-in-University_JACH_2012.pdf.

Urquant, B. y Pooley, J. (2007). The transition experience of Australian students to university: The importance of social support. The Australian Community Psychologist, 19(2), 78-91. Disponible en línea: http://groups.psychology.org.au/Assets/Files/19(2)_07_Pooley.pdf.

Van Tilburg, M., Vingerhoets, A. y Van Heck, G. (1997). Coping with homesickness: The construction of the Adult Homesickness Coping Questionnaire. Journal of Personality and Individual Differences, 22, 901-907. doi:10.1016/S01918869(97)00010-X.

Van Tilburg, M., Vingerhoets, A. y Van Heck, G. (1999). Determinants of homesickness chronicity: Coping and personality. Personality and Individual Differences, 27(3), 531-539. doi: 10.1016/S0191-8869(98)00262-1.

Verschuur, M. y Eurelings-Bontekoe, E. (2003). Homesickness, temperament and character. Personality and Individual Differences, 35, 757-770. doi: 10.1016/S0191-8869(02)00281-7.

Watt, S. y Badger, A. (2009). Effects of social belonging on homesickness: An application of the belongingness hypothesis. Personality and Social Psychology Bulletin, 35(4), 516-530. doi: 10.1177/0146167208329695. 